\title{
Triamcinolone acetonide injections for the treatment of recalcitrant post-radical prostatectomy vesicourethral anastomotic stenosis: A retrospective look at efficacy and safety
}

Sarah Neu${ }^{1}$; Humberto Vigil ${ }^{1}$; Jennifer A. Locke ${ }^{1}$; Sender Herschorn ${ }^{1}$

Division of Urology, Sunnybrook Health Sciences Centre, University of Toronto, Toronto, ON, Canada

Cite as: Neu S, Vigil H, Locke JA, et al. Triamcinolone acetonide injections for the treatment of recalcitrant post-radical prostatectomy vesicourethral anastomotic stenosis: A retrospective look at efficacy and safety. Can Urol Assoc J 2020 August 7; Epub ahead of print.

http://dx.doi.org/10.5489/cuaj.6644

Published online August 7, 2020

$* * *$

\section{Abstract}

Introduction: We aimed to evaluate the success of bladder neck injections of triamcinolone at the time of transurethral bladder neck incision (BNI) for prevention of recurrent vesicourethral anastomotic stenosis (VUAS) following prostate cancer treatment.

Methods: This is a retrospective cohort study examining patients with recurrent VUAS post-RP \pm radiation treated with triamcinolone injections at the time of BNI. VUAS was diagnosed by symptoms followed by cystoscopy or urethrography. The outpatient procedures were done under general anesthesia. Cold knife incisions were made at the three, nine, and 12 o'clock BN positions, followed by triamcinolone injections $(4 \mathrm{mg} / \mathrm{mL})$ into the three and nine o'clock incision sites. Treatment outcomes were determined with cystoscopy.

Results: Eighteen men underwent 25 procedures over a four-year period. Median age at diagnosis of VUAS was 65 (interquartile range [IQR] 61-68); median time to VUAS from RP was eight months (IQR 5-12). Fourteen patients (78\%) had radiation treatment. The cohort had 128 unsuccessful VUAS treatments, with a median of five failed treatments per patient (IQR 310). Failed treatments included BN dilation, BNI, BN injection of mitomycin $\mathrm{C}$, and urethral stent placement. Success rate after a mean of 16.3 months (standard deviation [SD] 8.1) from the time of triamcinolone injection was $83 \%(15 / 18)$. Six patients went on to have successful incontinence surgery. Five patients $(28 \%)$ had treatment complications (bleeding, urinary tract infection, pain, and urinary extravasation). The three non-responders are stable and awaiting retreatment with triamcinolone injection.

Conclusions: Triamcinolone bladder neck injections for post-RP VUAS are a useful and safe treatment for recurrent stenosis. 


\section{Introduction}

Prostate cancer is the second most common malignancy in men, with one in nine men diagnosed during their lifetime. ${ }^{1}$ Survival rates with prostate cancer are high, with a five-year net survival rate of $98 \%$ for prostate cancer of all stages. ${ }^{2}$ Therefore, therapy for complications from treatment of prostate cancer are necessary to prevent long term morbidity.

One of the recognized complications of surgical treatment for prostate cancer is vesicourethral anastomotic stenosis (VUAS), previously referred to as bladder neck contracture. Often occurring within the first year after radical prostatectomy, this complication occurs secondary to tissue fibrosis, with an increase in collagen deposition leading to scarring and contracture. $^{3,4}$ The incidence of VUAS is estimated at $4-29 \%$ following open-RP, compared to the decreased incidence of $0-1.4 \%$ following robotic-assisted laparoscopic prostatectomy (RALP). ${ }^{5-8}$ Open-RP continues to be a common treatment option for prostate cancer in Canada, and morbidity includes urinary tract infection (UTI), urinary retention, dysuria, incontinence, and an overall decrease in quality of life. Thus, VUAS treatment options are necessary.

Treatment options for VUAS vary, and include bladder neck dilation, bladder neck incision (BNI), trans-urethral resection, urethral stent, open bladder neck repair, chronic catheterization or supra-pubic (SP) tube, and urinary diversion. ${ }^{4,9,10}$ Endoscopic adjunct treatments include injection of mitomycin $\mathrm{C}(\mathrm{MMC})$ or corticosteroids, the most common being triamcinolone acetate. Triamcinolone works by enhancing collagenase activity to break down collagen and allows for tissue re-modelling. ${ }^{11}$

The outcomes of triamcinolone injections have not been robustly investigated, but studies have shown success rates as high as $83 \%$ in recurrent VUAS. ${ }^{12}$ The objective of this study is to evaluate the efficacy and safety of triamcinolone injections during BNI for recurrent or recalcitrant VUAS following open-RP in a contemporary series.

\section{Methods}

Research ethics board approval was obtained prior to initiation of this study.

\section{Patients}

A total of 18 men with recurrent or recalcitrant VUAS underwent BNI followed by triamcinolone injections between 2016-2019. Data was collected retrospectively. VUAS was diagnosed by voiding symptoms (urinary retention, slow urinary stream, or straining to empty), with or without urinary incontinence, followed by urethrography and cystoscopy with the inability to pass a 17F flexible cystoscope. Patients were followed with cystoscopy, urinary flow rates and post void residuals (PVR). Recurrent or recalcitrant VUAS was diagnosed by evidence of stenosis on cystoscopy following one or more VUAS treatments. Failed treatments in this cohort included bladder neck dilation, BNI, injection of MMC into the scar, or ALLIUM stent placement. Data collection included patient characteristics, prostate cancer pathology and treatment, VUAS management, continence status, triamcinolone treatments, incontinence 
treatments, and follow-up time, where available. Recurrent VUAS treatment decisions were made on a patient-by-patient basis. Treatment success was defined as the ability to easily pass a $17 \mathrm{~F}$ flexible cystoscope through the vesicourethral anastomosis at the three-month follow-up cystoscopy, without recurrence during the study period, with a mean follow-up time of 16.3 months (SD 8.1).

\section{Surgical technique}

All patients gave informed consent for treatment. The procedure was done under a general anesthesia in the lithotomy position. Antibiotic prophylaxis was administered. An incision at the bladder neck was made using a half-moon cold knife at the three, nine, and 12 o'clock positions until soft tissue and bleeding were encountered. A Triamcinolone vial of $40 \mathrm{mg} / \mathrm{mL}$ was diluted to $4 \mathrm{mg} / \mathrm{mL}$ with normal saline and the entirety of the solution was injected into the three and nine o'clock incision sites, using one to two punctures per incision site. An $18 \mathrm{~F}$ foley catheter was left in situ for 5-7 days. Antibiotics were given around the time of catheter removal. Patients with recent positive urine cultures or cloudy appearing urine received five days of antibiotics from post-operative day zero.

\section{Data analysis}

The primary outcome was resolution of the recurrent or recalcitrant VUAS after triamcinolone injection. Secondary outcomes included post-operative complications and success of subsequent incontinence surgery. The patient cohort and outcomes were described using descriptive statistics and expressed as mean and standard deviations (SD) for normally distributed continuous variables, median and interquartile range (IQR) for non-normally distributed continuous variables, and frequency and percentage for categorical variables. The Wilcoxon signed rank sum test was used to compare paired data. A p value of $<0.05$ was considered to be statistically significant. All data analysis was generated using SAS University (SAS Institute Inc).

\section{Results}

\section{Demographics}

A total of 18 patients were treated with 25 BNI's and triamcinolone injections between 20162019. The median age at diagnosis of VUAS was 65 years (IQR: 61-68). In this cohort 2/18 patients $(11 \%)$ were smokers and $4 / 18$ patients $(22 \%)$ had diabetes. All patients had an open-RP between $2005-2015,15 / 18(83 \%)$ had an open-RP as their primary treatment for prostate cancer, and 3/18 (17\%) had a salvage open-RP following primary radiation treatment. On post-operative specimens, grade group two was the most common pathology $(5 / 18,28 \%)$. Pathologic TNM staging was pT2 in 7/18 patients (38\%), and pT3 in 4/18 (22\%). Surgical margins were positive in $6 / 18$ patients $(33 \%)$. A total of $14 / 18(78 \%)$ patients received some form of radiation - ten patients received salvage radiation, three received radiation as primary prostate cancer treatment, 
and one received adjuvant radiation. Androgen deprivation therapy (ADT) was used in 5/18 patients $(28 \%)$ [TABLE 1$]$.

\section{Prior treatments for VUAS}

The median time to stenosis post-open RP was eight months (IQR: 5-12). Among the 18 patients 128 treatment failures were recorded, including 37 bladder deck dilations, 88 BNI's, two MMC injections, and one ALLIUM stent placement and subsequent removal. Tapered selfcatheterization was used in 7/18 (39\%) patients prior to triamcinolone treatment. The median number of treatment failures per patient was five (IQR: 3-10) [FIGURE 1].

\section{Efficacy}

Time from initial diagnosis of recurrent/recalcitrant VUAS to treatment with triamcinolone injection was between 11-127 months, with a mean of 48 months (SD 34.6). Triamcinolone injections were successful in treating recurrent/recalcitrant VUAS in $15 / 18$ patients $(83 \%)$ [FIGURE 2]. Patients received a median of 1 treatment (IQR 1-2) for success, with 9/18 patients $(50 \%)$ requiring one treatment, 5/18 (28\%) required two treatments, and $1 / 18(6 \%)$ requiring three treatments; median time between injections was four months (IQR: 3-10). Follow-up with initial post-treatment cystoscopy was at a median of two months (IQR 2.0-2.5 months), although treatment was only considered successful at the three-month cystoscopy or beyond. Patients had a mean follow-up time of 16.3 months (SD 8.1) [TABLE 2].

Eight patients $(44 \%)$ were in urinary retention prior to triamcinolone treatment. The median post-operative PVR was at 0cc (IQR 0-0, range 0-16cc) at their first follow-up visit. For those who were not in urinary retention, the median post-operative Qmax was higher than the median pre-operative Qmax, at $20 \mathrm{cc} / \mathrm{second}$ compared to $10.5 \mathrm{cc} / \mathrm{second}$, although not statistically significant $\mathrm{p}=0.08$.

Of the three treatment non-responders, two had undergone salvage radiation therapy. Two patients received one triamcinolone injection each, and one patient had received two triamcinolone injections, to date. At the time of this study all three patients had stable urinary symptoms, without urinary catheter use, and were awaiting subsequent BNI with triamcinolone injection.

The patient with the greatest number of failed treatments had had 25 VUAS treatments since his open-RP in 2010. He required approximately three treatments per year. This patient underwent three triamcinolone injection treatments, at three and four months apart. Since his last injection the patient has not had a recurrence of his VUAS in 24 months.

\section{Complications}

Clavien-Dindo grade I complications were noted in 5/18 patients $(28 \%)$. One patient was admitted overnight for monitoring of gross hematuria. One patient was re-admitted for pubic pain thought to be from urinary extravasation two weeks after his catheter had been removed (on 
post-operative day seven). This resolved with catheter drainage for three weeks and antibiotics. One patient developed a culture proven UTI. One patient developed self-resolving post-operative pain, and one patient went into post-operative urinary retention, which resolved. No side effects due to drug interactions were noted.

\section{Incontinence}

Incontinence was present in 11/18 (61\%) of patients pre-operatively, and six of these patients went on to have successful incontinence surgery - five artificial urinary sphincters and one ATOMS sling placement, without a recurrence of VUAS. Of the remaining five patients two are continuing VUAS treatment for recurrent stricture, one is awaiting incontinence surgery, one developed metastatic prostate cancer and elected to avoid further surgery, and one was lost to follow-up. The median time from successful triamcinolone treatment to incontinence surgery was 12 months (IQR 10-18).

\section{Discussion}

The optimal treatment for VUAS is unknown. The Société Internationale d'Urologie and the International Consultation on Urological Diseases (SIU/ICUD) suggest that treatment of VUAS follow a graded approach, beginning with endourologic procedures. ${ }^{9}$ LaBossiere et. al. reviewed 142 patients with VUAS and found the success rate of one endoscopic procedure to be $44 \%{ }^{13}$ For those who fail initial endoscopic treatment, many go on to have multiple endoscopic treatments without success (our study showing a median of five treatments per patient). Alternative treatments then include urethral stents, MMC injections, open or robotic reconstruction, urinary diversion, or chronic indwelling catheter, SP tube, or self-catheterization. Compared to triamcinolone injections, the majority of these options come with the potential for high morbidity. Urethral stents have proven success but come with the risk of tissue intrusion and bladder migration. ${ }^{9}$ A 2017 study of MMC injection into the bladder neck concluded that it is a safe and effective treatment for recurrent VUAS, with a success rate of $86 \%$ with no reported adverse events. ${ }^{14}$ This is in contrast to the larger 2015 TURNS study, which showed a 75\% success rate, but serious adverse events - including osteitis pubis, rectourethral fistula, and bladder neck necrosis; three of these patients required urinary diversion with cystectomy. ${ }^{15}$ However, limitations of the TURNS study included the use of various MMC doses and injection techniques, with short-term follow-up. At our centre, MMC costs $\$ 195$ CND per 20mg vial, compared to triamcinolone, which costs $\$ 8.96 \mathrm{CND}$ per $40 \mathrm{mg} / \mathrm{mL}$ vial. This large cost saving, with seemingly minimal side effects, should be taken into consideration.

Open reconstruction for VUAS remains an option. Open perineal approach, with or without combined abdominoperinal access, have shown high success rates, but at the cost of high incontinence rates. The use of robotic approach to bladder neck reconstruction is promising, with a $75-100 \%$ success rate with minimal incontinence. ${ }^{16-18}$ 
The results of this series are in keeping with the findings of efficacy and safety in previously published studies of steroid injections. ${ }^{11,12,19,20}$ Eighty-three percent were VUASfree at a median of 16.3 months. The minor complications that did occur are in keeping with BNI-associated risks rather than triamcinolone-specific morbidity. No patients had any long-term complications at the time of completion of this study.

One factor playing a role in the success of this procedure may be due to the use of deep lateral incisions at the bladder neck. The success rate for deep lateral BN incisions in refractory VUAS in the literature ranges from $72 \%-80 \%,{ }^{21-23}$ similar to our success rate of $83 \%$ with deep lateral incisions with triamcinolone injection. Our patient cohort had a total of 88 BNI's prior to triamcinolone treatment. Of these, 14 were done at our centre using a deep lateral incision method, and still went on to develop recurrent VUAS. It is uncertain whether the remaining BNI's done at outside centres were done using this method. A trial comparing BNI with deep lateral incisions compared to triamcinolone injection without deep lateral incision has not yet been done. Other factors effecting success of triamcinolone therapy may be prior MMC treatment, which may have made the triamcinolone injections more successful. The effect of $\mathrm{MMC}$ in conjunction with triamcinolone is not known.

The appropriate timing for surgical management of incontinence post-VUAS treatment in the form of AUS or male slings is unknown. One study describing management of VUAS with holmium: yttrium-aluminum-garnet (Ho:YAG) laser incision with triamcinolone injections implanted an AUS in 11 patients 8-10 weeks post-procedure; of these, two eroded and one was explanted for malfunction. ${ }^{12}$ Studies of AUS insertion three months following BNI treatment alone for VUAS at have a reported success rate of $88 \%$; one patient had sphincter erosion and the second required a secondary sling procedure for ongoing incontinence. ${ }^{21}$ In our study we show six patients who went on to have successful perineal incontinence surgery, without complication, at a median of 12 months after recurrent VUAS treatment with BNI and triamcinolone injection.

For clinicians embarking on the use of triamcinolone injections for VUAS treatment, we recommend first follow-up cystoscopy at two months post-procedure, or earlier if symptoms recur.

Limitations to this study include its relatively small sample size. It is a retrospective case series without a control group. Additionally, we did not investigate asymptomatic VUAS, and only patients with urinary symptoms in keeping with VUAS were investigated with cystoscopy or urethrography. Furthermore, triamcinolone injections were delivered by a single surgeon at a single institution. Prospective trials of triamcinolone injections are needed to elucidate the full treatment effect. 


\section{Conclusions}

This study adds to the body of evidence that triamcinolone injections are a safe and effective alternative treatment for recurrent and recalcitrant VUAS after radical prostatectomy. Prolonged follow-up for recurrence is still required.

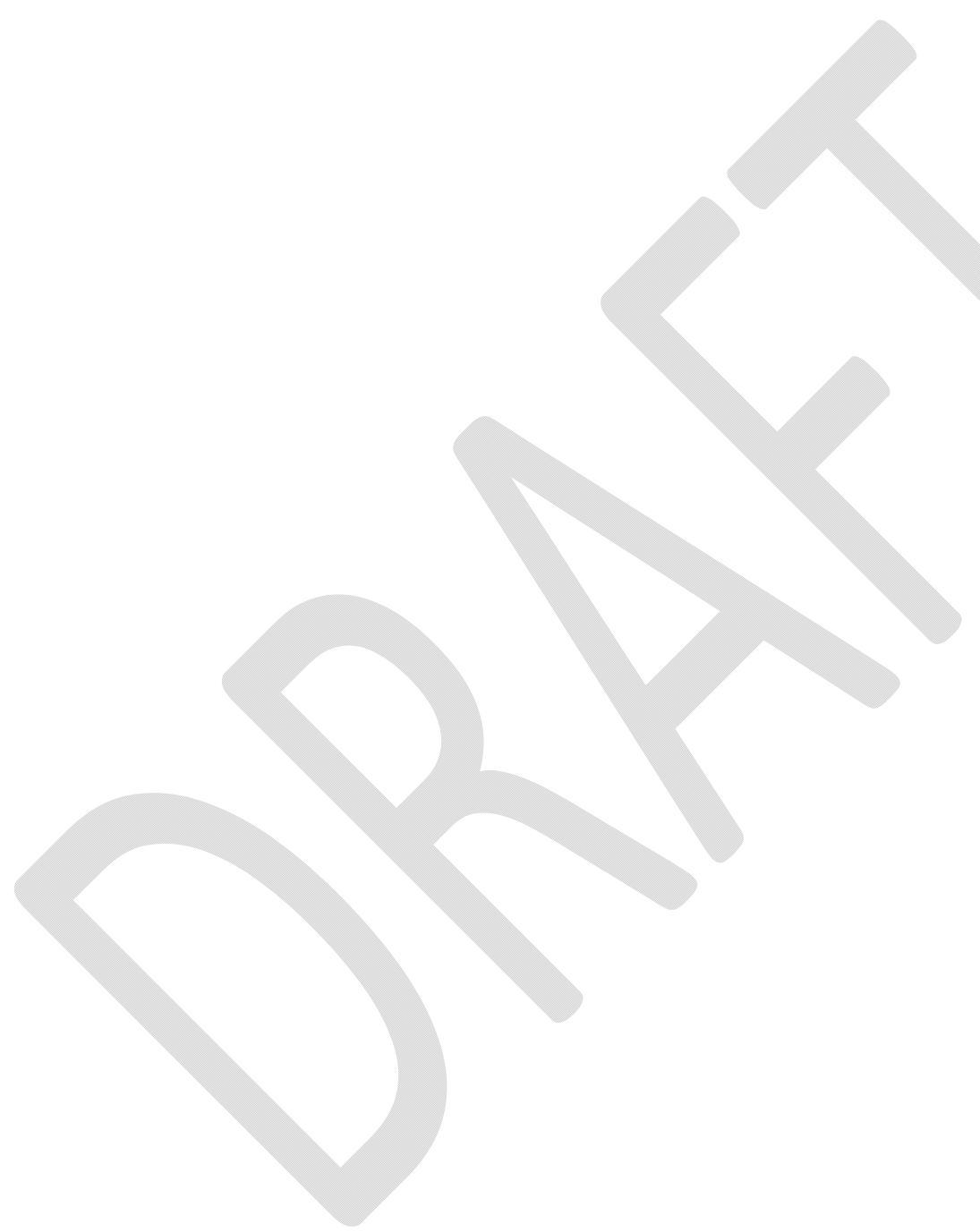




\section{References}

1. About Prostate Cancer. [Prostate Cancer] 2019 July 24, 2019]; Available from: https://www.cancer.org/content/dam/CRC/PDF/Public/8793.00.pdf

2. Noone AM, Howlader N, Krapcho M, et al. SEER Cancer Statistics Review April 2018: National Cancer Institute

3. Giannarini G, Manassero F, Mogorovich A, et al. Cold-knife incision of anastomotic strictures after radical retropubic prostatectomy with bladder neck preservation: efficacy and impact on urinary continence status. Eur Urol 2008;54:647-56.

4. Nicholson HL, Al-Hakeem Y, Maldonado JJ, et al. Management of bladder neck stenosis and urethral stricture and stenosis following treatment for prostate cancer. Transl Androl Urol 2017;S92-102.

5. Breyer BN, Davis CB, Cowan JE, et al. Incidence of bladder neck contracture after robotassisted laparoscopic and open radical prostatectomy. BJU Int 2010; 106:1734-8.

6. Carlsson S, Nilsson AE, Schumacher MC, et al. Surgery-related complications in 1253 robot-assisted and 485 open retropubic radical prostatectomies at the Karolinska University Hospital, Sweden. Urology 2010; 75:1092-7.

7. Coelho RF, Palmer KJ, Rocco B, et al. Early complication rates in a single-surgeon series of 2500 robotic-assisted radical prostatectomies: report applying a standardized grading system. Eur Urol 2010; 57:945-52.

8. Webb DR, Sethi K, and Gee K. An analysis of the causes of bladder neck contracture after open and robot-assisted laparoscopic radical prostatectomy. BJU Int 2009; 103:95763.

9. Herschorn S, Elliott S, Coburn M, et al. SIU/ICUD Consultation on Urethral Strictures: Posterior Urethral Stenosis After Treatment of Prostate Cancer. Urology 2014;83:S59-70.

10. Pfalzgraf D, Siegel F, Kriegmair MC, et al. Bladder Neck Contracture After Radical Prostatectomy: What Is the Reality of Care? J Endourol 2017; 31:50-6.

11. Damico CF, Mebust WK, Valk WL, et al. Triamcinolone: adjuvant therapy for vesical neck contractures. J Urol 1973; 110:203-4.

12. Eltahawy E, Gur U, Virasoro R, et al. Management of recurrent anastomotic stenosis following radical prostatectomy using holmium laser and steroid injection. BJU Int 2008; 102:796-8.

13. LaBossiere JR, Cheung D, and Rourke K. Endoscopic Treatment of Vesicourethral Stenosis after Radical Prostatectomy: Outcomes and Predictors of Success. J Urol 2016; 195:1495-1500.

14. Sourial MW, Richard PO, Bettez M, et al. Mitomycin-C and urethral dilatation: A safe, effective, and minimally invasive procedure for recurrent vesicourethral anastomotic stenoses. Urol Oncol 2017; 35:672.e15-19.

15. Redshaw JD, Broghammer JA, Smith TG, et al. Intralesional injection of mitomycin C at transurethral incision of bladder neck contracture may offer limited benefit: TURNS Study Group. J Urol 2015; 193:587-92.

16. Kirshenbaum EJ, Zhao LC, Myers JB, et al. Patency and Incontinence Rates After Robotic Bladder Neck Reconstruction for Vesicourethral Anastomotic Stenosis and 
Recalcitrant Bladder Neck Contractures: The Trauma and Urologic Reconstructive Network of Surgeons Experience. Urology 2018; 118:227-33.

17. Granieri MA, Weinberg AC, Sun JY, et al. Robotic Y-V Plasty for Recalcitrant Bladder Neck Contracture. Urology 2018; 117:163-5.

18. Musch M, Hohenhorst JL, Vogel A, et al. Robot-assisted laparoscopic Y-V plasty in 12 patients with refractory bladder neck contracture. J Robot Surg 2018; 12:139-45.

19. Bannow JE, Mebust WK, Foret JD, et al. A second look at injection of vesical neck contractures with triamcinolone. J Urol 1976; 116:39-40.

20. Farah RN, DiLoreto RR, and Cerny JC. Transurethral resection combined with steroid injection in treatment of recurrent vesical neck contractures. Urology 1979; 13:395-7.

21. Brede C, Angermeier K, and Wood H. Continence outcomes after treatment of recalcitrant postprostatectomy bladder neck contracture and review of the literature. Urology 2014; 83:648-52.

22. Gousse AE, Tunuguntla HS, and Leboeuf L. Two-stage management of severe postprostatectomy bladder neck contracture associated with stress incontinence. Urology 2005; 65:316-9.

23. Ramirez D, Zhao LC, Bagrodia A, et al. Deep lateral transurethral incisions for recurrent bladder neck contracture: promising 5 -year experience using a standardized approach. Urology 2013; 82:1430-5.

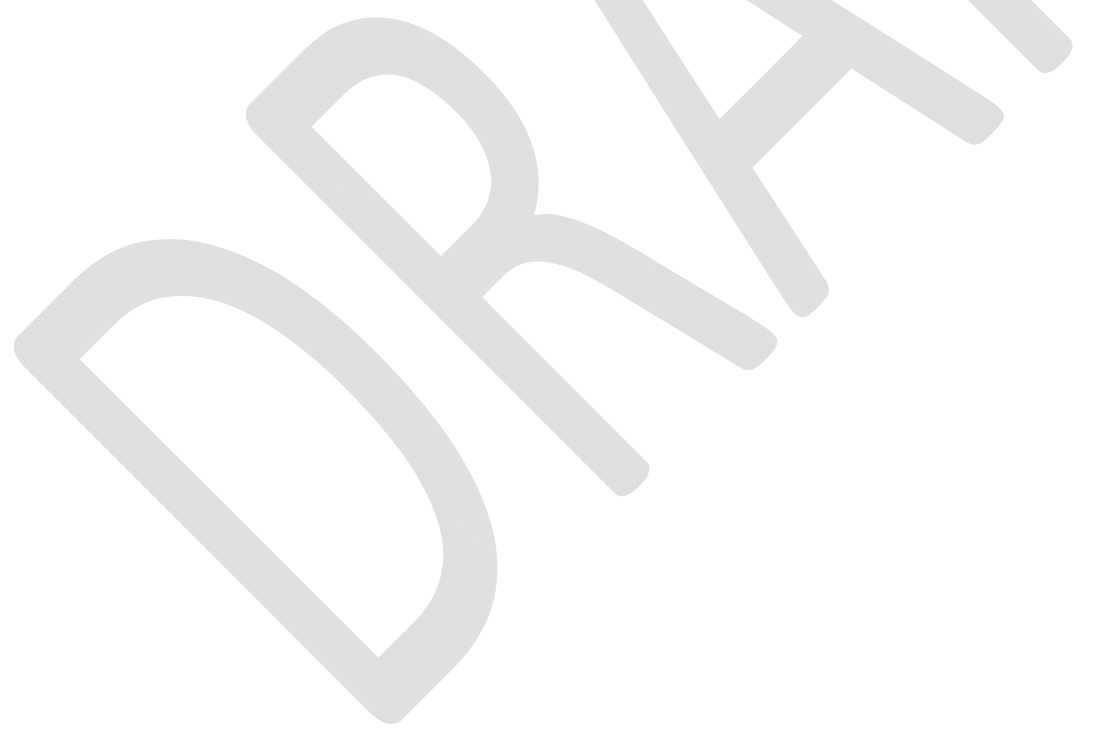




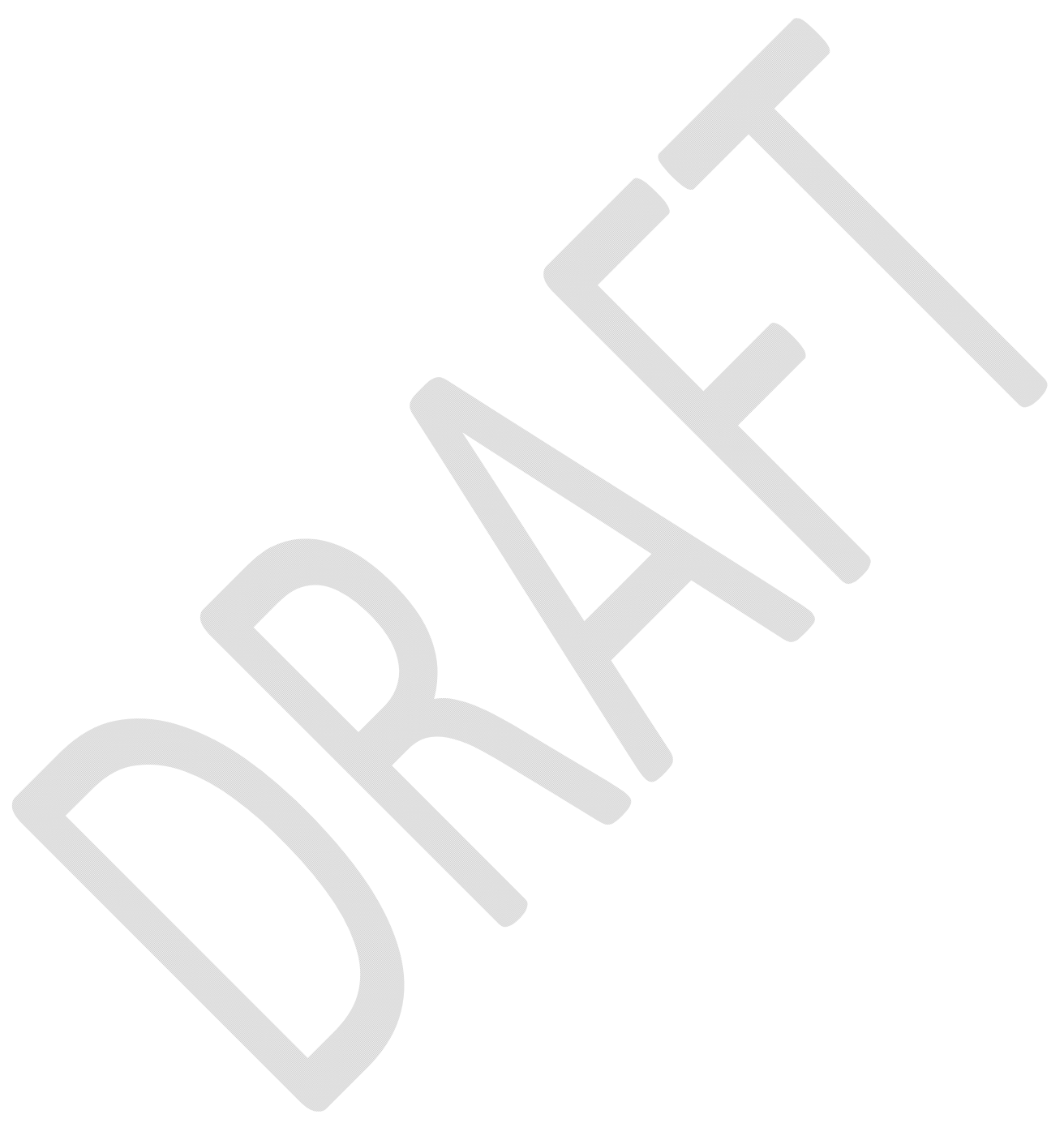




\section{Figures and Tables}

Fig. 1. Failed vesicourethral anastomotic stenosis (VUAS) treatments.

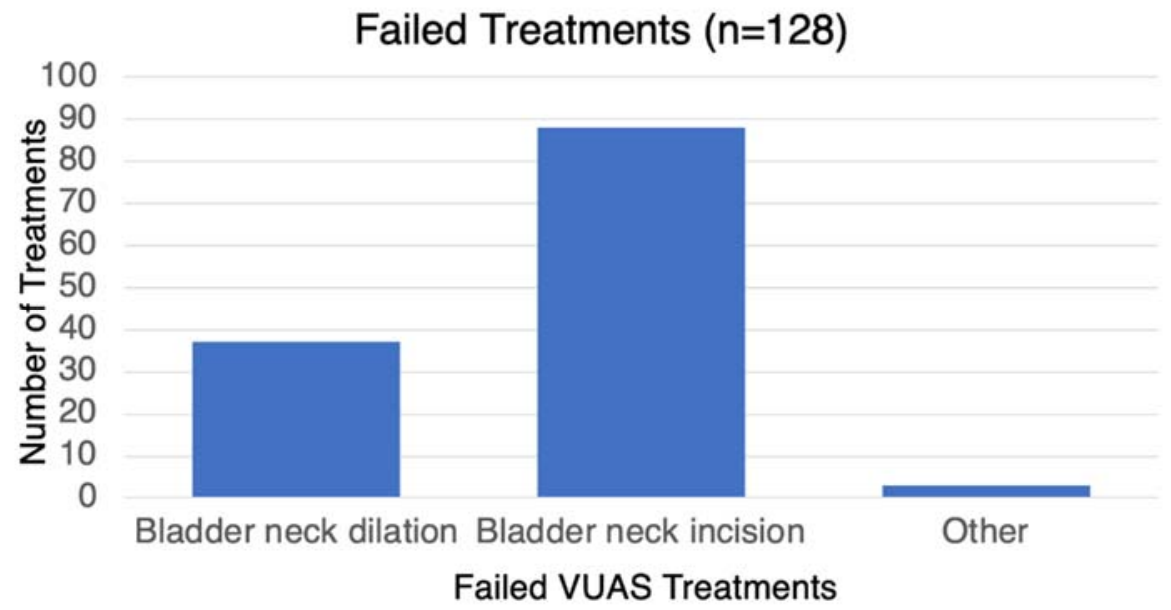

Fig. 2. Number of failed vesicourethral anastomotic stenosis (VUAS) treatments per patient.

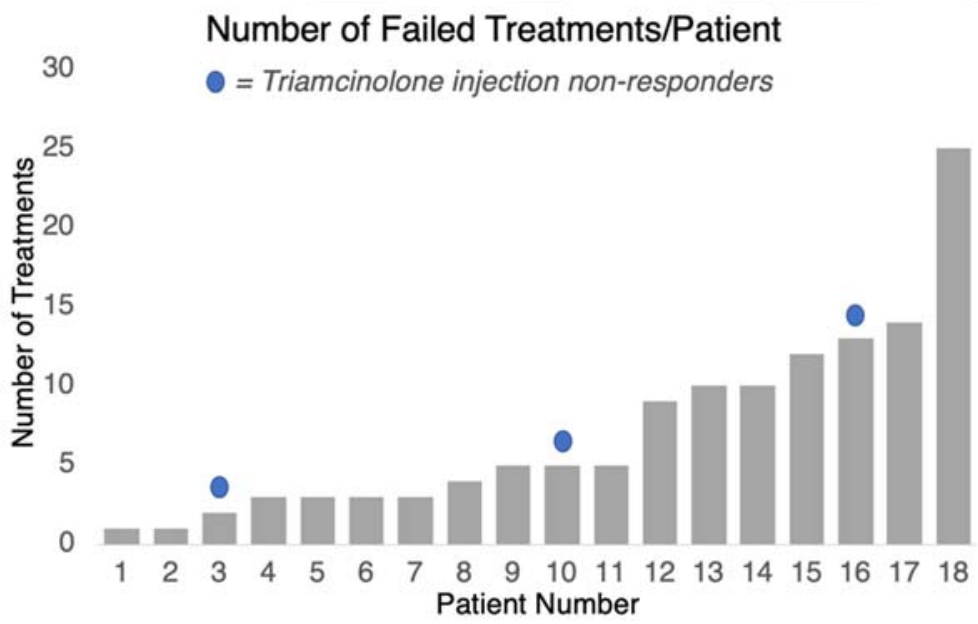




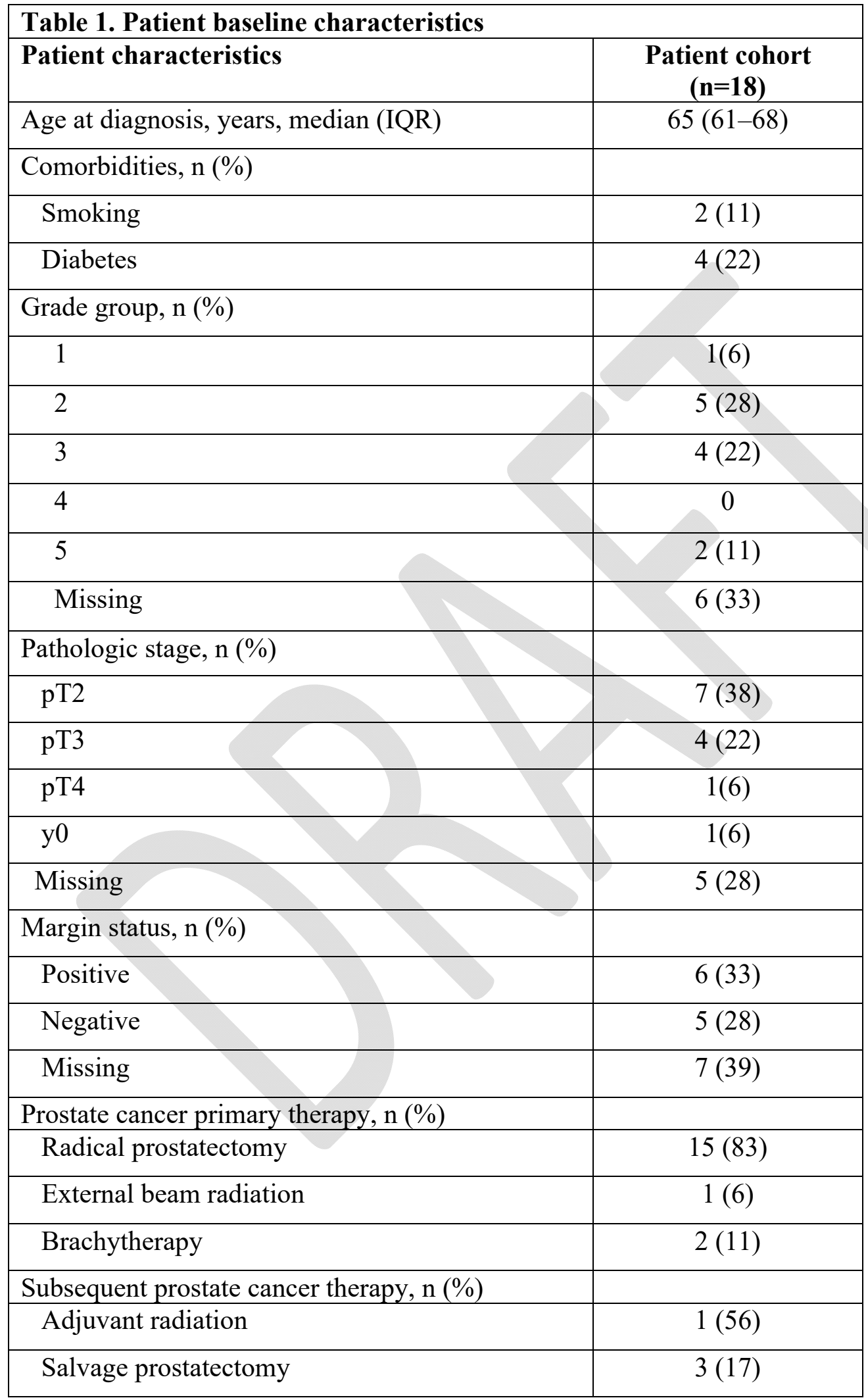




\begin{tabular}{|l|c|}
\hline Salvage radiation & $10(56)$ \\
\hline ADT & $5(28)$ \\
\hline
\end{tabular}

ADT: androgen deprvation therapy.

\section{Table 2. Triamcinolone treatment characteristics}

\begin{tabular}{|l|c|}
\hline Characteristics & Participants (n=18) \\
\hline $\begin{array}{l}\text { Time from VUAS diagnosis to triamcinolone } \\
\text { treatment (months), mean (SD) }\end{array}$ & $48(34.6)$ \\
\hline $\begin{array}{l}\text { Number of triamcinolone treatments per patient, } \\
\text { median (IQR) }\end{array}$ & $1(1-2)$ \\
\hline Success of triamcinolone, n (\%) & $15(83)$ \\
\hline Non-responders, n (\%) & $3(17)$ \\
\hline Followup (months), mean (SD) & $16.3(8.1)$ \\
\hline
\end{tabular}

IQR: interquartile range; SD: standard deviation; VUAS: vesicourethral anastomotic stenosis. 